\title{
Numerical Modelling and Design of an Eddy Current Sensor
}

\author{
Philip May ${ }^{1}$ and Erping Zhou ${ }^{2}$ \\ ${ }^{1}$ Elcometer Ltd. and \\ 2University of Bolton
}

UK

\section{Introduction}

Eddy current testing involves exciting a coil with a fixed frequency or pulse and bringing it into close proximity with a conductive material. The electrical impedance of the coil changes due to the influence of electrical 'eddy currents' in the material. Using an eddy current technique, the sizing of surface and sub-surface defects, measurements of thickness of metallic plates and of conductive and non-conductive coatings on metal substrates, assessment of corrosion, ductility, heat treatment and measurements of electrical conductivity and magnetic permeability are all possible and quantifiable. The eddy current method has become one of the most successful non-destructive techniques for testing conductive coatings on conductive substrates.

The data acquired from eddy current sensors however is affected by a large number of variables, which include sample conductivity; permeability; geometry and temperature as well as sensor lift off. The multivariable properties of sample coatings add an even greater level of complexity. Many of these problems have been overcome in the laboratory using precision wound air-cored coils, multiple excitation frequencies and theoretical inversion models. High levels of agreement between theoretical models and measurement however are only possible with accurately constructed coils, which are difficult to manufacture in practice. Coils are also prone to poor sensitivity, poor resolution, and a poor dynamic range as well as self-resonance at high frequencies, which make them unsuitable for online process control. Many of the problems associated with air-cored coils however can be overcome when the coils use ferrite cores or cup cores.

Inversion models often make use of simplifying assumptions, which include symmetrically wound coils, constant current distributions in coil regions and ideal test materials. Ideal coils simply do not exist outside the laboratory; ideal test materials do not exist outside the laboratory either. An example of non-ideal test materials is hot-dip galvanising, where molten zinc reacts with steel to form distinct eutectic alloy layers (Langhill, 1999). Another example is case hardening in steel. Steel also has a magnetic permeability that is frequency dependent and subject to localised variation (Bowler, 2006).

Other than non-ideal coils and test materials, a practical limit exists to the information that can be extracted through eddy current testing (Norton \& Bowler, 1993). Eddy currents can 
only really sense the presence of layer boundaries owing to the integrating character of eddy current signals. Glorieux and co-workers give an example of this, observing that sharp material profile features appear smooth under reconstruction (Glorieux et al, 1999). Another limitation, which affects coatings on steel, is the permeability-conductivity ratio and coating conductivity-thickness product (Becker et al, 1988). One of these quantities must be known prior to inspection.

This chapter focuses on the development and testing of a new highly accurate and highly sensitive ferrite-cored sensor and a novel magnetic moment model of the sensor, which requires only the discretisation of the sensor core-air boundary interface. The chapter starts by developing a set of partial differential equations (PDE) to model the vector potential fields present in the regions bounding the sensor. Sensor regions were considered to be source-less with imaginary surface currents imposed at region interfaces. Green's functions were determined for all bounded regions. Basis functions were then used to represent the sensor cores surface current distribution, which were then formed into a set of $2 \mathrm{~N}$ linearly independent equations by applying the relevant boundary conditions. A matrix method was finally developed to solve these equations using a moment method.

The matrix method was further developed in this chapter in order to calculate sensor coil impedance and induced voltage. An efficient material profile function $m(\alpha)$ for modelling the interaction between the sensor and test material was also developed and verified. A novel form of parameterisation was adopted for $m(\alpha)$. The accuracy and convergence of the vector potentials generated by the source coil and core-air boundary surface currents was reviewed and a new free-space Green's function introduced.

\section{Sensor theoretical model}

This section introduces a new ferrite-cored eddy current sensor and develops integral equations to characterise the source vector potential and core vector potential fields. Closed form solutions of the core equations are applied to the core-air boundary interface, generating $2 \mathrm{~N}$ linearly independent equations with $2 \mathrm{~N}$ unknown coefficients. The unknown coefficients are evaluated using the method of weighted residuals.

\subsection{Basic sensor design}

When a ferrite core is used in an eddy current sensor, the coil inductance, sensitivity and resolution increase significantly (Blitz, 1991, Moulder et al, 1992). A ferrite core is therefore incorporated into the sensor design used for this chapter, which is shown in figure 1. Coaxial to the ferrite core below are three coils, a central source coil, which carries a current I amps and two sense or pick-up coils. The sensor is a reflection sensor (or transformer style sensor) with pick-up coils in a differential configuration. Each coil is assumed to have $n_{\mathrm{c}}$ coil turns per unit area with a total of $N_{c}$ turns. The sensor is located in free space and positioned above and orthogonal to a medium, which is comprised of $M$ planar layers. Each layer is considered to be linear, isotropic and homogeneous, where the $i^{\text {th }}$ layer has conductivity $\sigma_{\mathrm{i}}$ and permeability $\mu_{\mathrm{i}}$. 


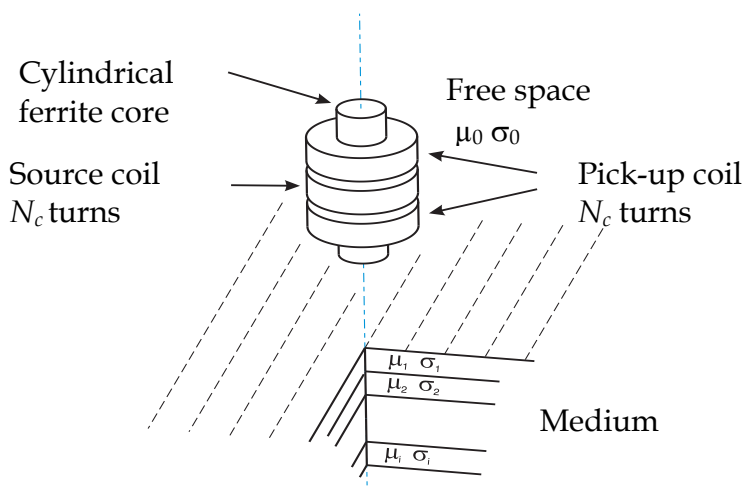

Fig. 1. Ferrite-Cored Eddy Current Sensor.

Certain assumptions are made about the sensor, which are listed below:

- The self-resonant frequency of each coil is greater than the maximum operating frequency of the sensor as a measuring system; corrections for coil self-capacitance or coil-ferrite capacitance is not considered necessary (Harrison et al, 1996).

- The source coil is considered to be a region of constant current density.

- Pick-up coils are matched and act into loads of infinite impedance. Pick-up coils generate no magnetic flux.

- The sensor core is soft magnetic ferrite. The core is assumed to be linear, isotropic and homogeneous; core conductivity is assumed to be negligible.

In order to begin an analysis of the sensor of Fig. 1, the ferrite core and pick-up coils were removed and the source coil replaced with a delta function coil. The free space region bounding the sensor was also divided into two regions, one above the plane of the delta function coil (region 1) and one below and extending to the surface of the medium (region 2). See figure 2.

Using Maxwell's equations and the homogeneous wave equation, the PDE defining the source vector potential field $A_{S}$ in any of the regions of figure 2 is of the form:

$$
\nabla^{2} A_{S}=\mu J_{t}
$$

$\mu$ represents medium permeability and $J_{t}$ is the total electric current density. If $J_{t}$ is comprised of an impressed current density $J_{s}$ and an effective electric conduction current density $J_{c e}$, then

$$
\nabla^{2} \boldsymbol{A}_{\boldsymbol{S}}=\mu\left(\boldsymbol{J}_{\boldsymbol{s}}+\boldsymbol{J}_{\boldsymbol{c e}}\right)
$$




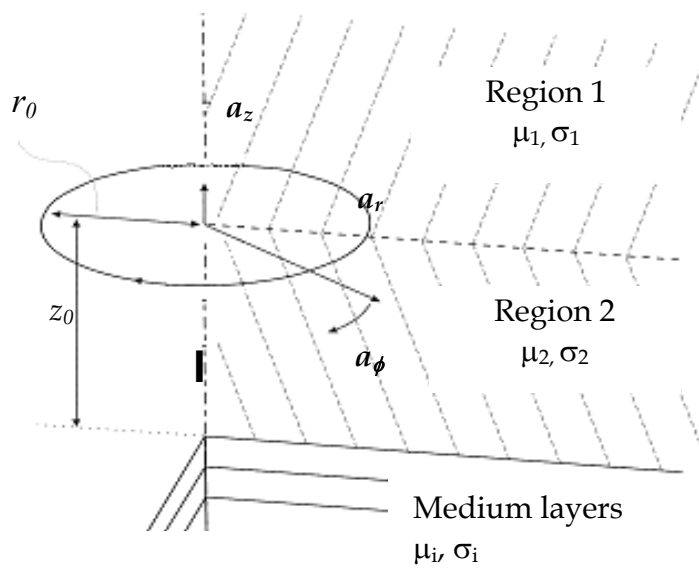

Fig. 2. Delta Function Source Coil Located above a Layered Medium.

If a delta function coil $\boldsymbol{I}_{\boldsymbol{0}}=\delta\left(r-r_{0}\right) \delta\left(z-z_{0}\right) \boldsymbol{a}_{\boldsymbol{\varphi}}$ is located at $\left(r_{0}, z_{0}\right)$, where

$$
\delta\left(r-r_{0}\right) \delta\left(z-z_{0}\right)=\left\{\begin{array}{l}
\infty, \quad \text { if } \quad\left(r=r_{0}\right), \quad\left(z=z_{0}\right) \\
0 \quad \text { otherwise }
\end{array}\right.
$$

and current density $\boldsymbol{J}_{c e}=j \omega \mu \sigma \boldsymbol{A}_{S}$, then:

$$
\nabla^{2} \boldsymbol{A}_{\boldsymbol{S}}-j \omega \mu \sigma \boldsymbol{A}_{\boldsymbol{S}}+\mu \boldsymbol{I}_{\boldsymbol{0}}=0 .
$$

Using the vector identity $\nabla^{2} \boldsymbol{B}=\nabla(\nabla \cdot \boldsymbol{B})-\nabla \times \nabla \times \boldsymbol{B}$ gives

$$
\nabla\left(\nabla \cdot \boldsymbol{A}_{\boldsymbol{S}}\right)-\nabla \times \nabla \times \boldsymbol{A}_{\boldsymbol{S}}-j \omega \mu \sigma \boldsymbol{A}_{\boldsymbol{S}}+\mu \boldsymbol{I}_{\boldsymbol{O}}=0
$$

Since the coil excitation is azimuthal and since both the media and sensor core have axial symmetry, then vector potential $A_{s}$ will also be azimuthal, hence let the source field be

$$
\boldsymbol{A}_{\boldsymbol{S}}=A_{S \varphi}(r, z) \boldsymbol{a}_{\varphi} .
$$

Substituting the Coulomb gauge $\nabla \cdot \boldsymbol{A}_{\boldsymbol{S}}=0$ into PDE (5) gives

$$
-\nabla \times \nabla \times \boldsymbol{A}_{\boldsymbol{S}}-j \omega \mu \sigma \boldsymbol{A}_{\boldsymbol{S}}+\mu \boldsymbol{I}_{\boldsymbol{0}}=0
$$

and using: 


$$
\nabla \times \nabla \times \boldsymbol{A}_{\boldsymbol{S}}=\left|\begin{array}{ccc}
\boldsymbol{a}_{\boldsymbol{r}} / r & \boldsymbol{a}_{\boldsymbol{\varphi}} & \boldsymbol{a}_{z} / r \\
\partial / \partial r & \partial / \partial \phi & \partial / \partial z \\
-\partial A_{S \varphi} / \partial z & 0 & (1 / r) \partial\left(r A_{S \varphi}\right) / \partial r
\end{array}\right|
$$

gives the PDE for the delta function coil:

$$
\frac{\partial^{2} A_{s \varphi}}{\partial r^{2}}+\frac{1}{r} \frac{\partial A_{s \varphi}}{\partial r}-\frac{A_{s \varphi}}{r^{2}}+\frac{\partial A_{s \varphi}}{\partial z^{2}}-j \omega \mu \sigma A_{s \varphi}+\mu \delta\left(r-r_{0}\right) \delta\left(z-z_{0}\right)=0 .
$$

Equation (9) is widely recognised as the PDE first used by Dodd and Deeds (Dodd \& Deeds, 1968). If $G_{s}\left(r, z ; r_{0}, z_{0}\right)$ is the Green's function for equation (9), then:

$$
A_{s \varphi}(r, z)=\mu G_{S}\left(r, z ; r_{0}, z_{0}\right) .
$$

If the coil has a rectangular cross section and a source distribution $J_{s}=J_{s \varphi}\left(r_{0}, z_{0}\right) \boldsymbol{a}_{\varphi}$, then:

$$
A_{s \varphi}(r, z)=\mu \iint G_{s}\left(r, z ; r_{0}, z_{0}\right) J_{s \varphi}\left(r_{0}, z_{0}\right) d r_{0} d z_{0}
$$

The Green's function for equation (11) first proposed by Cheng and co-workers (Cheng et al, 1971) and forming the basis of nearly all subsequent eddy current research, is given below for the generalised $n^{\text {th }}$ media layer of figure 2:

$$
G_{s}^{(n)}\left(r, z ; r^{\prime}, z^{\prime}\right)=\int_{0}^{\infty}\left[B_{n}(\alpha) e^{-\alpha_{n} z}+C_{n}(\alpha) e^{\alpha_{n} z}\right] J_{1}(\alpha r) d \alpha
$$

$B_{n}(\alpha)$ and $C_{n}(\alpha)$ are media dependent functions and $J_{1}(\alpha r)$ is a Bessel function of the first order and first kind; $\alpha$ is defined for each region as follows:

$$
\alpha_{n}^{2}=\alpha^{2}+j \omega \mu_{n} \sigma_{n}
$$

\subsection{The influence of the sensor core}

The Cheng method imposes surface currents on media layer boundaries according to the surface equivalence theorem, where coefficients $B_{n}(\alpha)$ and $C_{n}(\alpha)$ are determined by enforcing boundary conditions at each layer interface. The sensor core can be treated in exactly the same way. Figure 3 shows surface current $J$ impressed on closed surface $S$ at the sensor core-air interface.

Figure 3 shows the sensor core partitioned into two separate regions, an external region (vector potential $A_{E}$ ) and an internal source-less region (vector potential $A_{I}$ ). Considering the internal region first, let a surface current $J_{I}$ be impressed on the closed surface $S$. Let $J_{I}$ be azimuthal, and at some arbitrary point $\rho^{\prime}$ on $S$, let the limiting value of $J_{I}$ be a delta function source: 


$$
\boldsymbol{J}_{\boldsymbol{I}}(\rho)=\int d^{3} \rho^{\prime} \delta\left(\rho-\rho^{\prime}\right) \boldsymbol{J}_{\boldsymbol{I}}\left(\rho^{\prime}\right)
$$

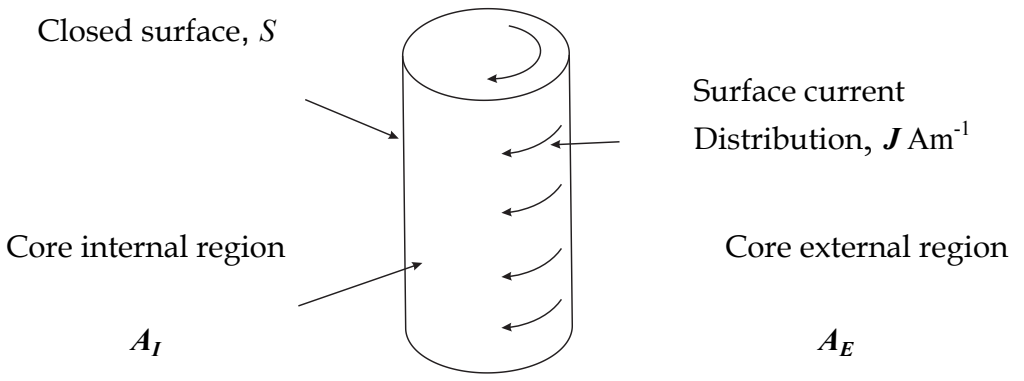

Fig. 3. Surface Current Distribution J on Core Surface $S$.

Since $\boldsymbol{J}_{\boldsymbol{I}}$ is azimuthal it follows that vector potential $\boldsymbol{A}_{\boldsymbol{I}}$ is likewise azimuthal, which leads To the following PDE for the component $A_{I \varphi}$ :

$$
\frac{\partial^{2} A_{I \varphi}}{\partial r^{2}}+\frac{1}{r} \frac{\partial A_{I \varphi}}{\partial r}-\frac{A_{I \varphi}}{r^{2}}+\frac{\partial A_{I \varphi}}{\partial z^{2}}+\mu_{I} \delta\left(r-r^{\prime}\right) \delta\left(z-z^{\prime}\right)=0
$$

with the solution for $J_{I}=J_{I \varphi}\left(r^{\prime}, z^{\prime}\right) a_{\varphi}$

$$
A_{I \phi}(r, z)=\mu_{I} \int_{C} G_{I}\left(r, z ; r^{\prime}, z^{\prime}\right) J_{I \varphi}\left(r^{\prime}, z^{\prime}\right) d s^{\prime}
$$

Integration is along a contour $C$ on closed surface $S . G_{I}\left(r, z ; r^{\prime}, z^{\prime}\right)$ and $G_{s}\left(r, z ; r_{0}, z_{0}\right)$ are clearly identical and differ only in media dependent functions $B_{n}(\alpha)$ and $C_{n}(\alpha)$. A similar set of equations does not directly follow for the external core field $A_{E}$ due to the presence of source current $J_{s}$. The field in this region must be regarded as the vector sum of the source field $A_{s}$ and a source-less scattered field $A_{R}$ (Yildir et al, 1992):

$$
A_{E}=A_{R}+A_{s}
$$

Concentrating on the source-less scattered field $A_{R}$ and impressing a scattering current $J_{R}$ on $S$, leads to the following:

$$
A_{R \varphi}(r, z)=\mu \int_{C} G_{R}\left(r, z ; r^{\prime}, z^{\prime}\right) J_{R \varphi}\left(r^{\prime}, z^{\prime}\right) d s^{\prime}
$$

$G_{R}\left(r, z, r^{\prime}, z^{\prime}\right)$ and $G_{S}\left(r, z ; r_{0}, z_{0}\right)$ are the same function as both are determined for the same $M$ +2 media layers. A solution to equation (18) proceeds by expanding surface current $J_{R \varphi}$ as follows (Balanis, 1989):

$$
J_{R \varphi}\left(r^{\prime}, z^{\prime}\right)=\sum_{i=1}^{N} u_{i} a_{i}\left(r^{\prime}, z^{\prime}\right)
$$


where $a_{i}$ represents a basis function and $u_{i}$ the basis function coefficient. Substituting equation (19) into (18) gives the following:

$$
A_{R \varphi}(r, z)=\mu \sum_{i=1}^{N} u_{i} \int_{C} G_{R}\left(r, z ; r^{\prime}, z^{\prime}\right) a_{i}\left(r^{\prime}, z^{\prime}\right) d s^{\prime}
$$

Basis functions were now chosen to accurately represent the anticipated unknown function $J_{R \varphi}$. A piecewise constant sub-domain function was chosen to do this, which is of the form shown below:

$$
\begin{aligned}
& a_{i}\left(r^{\prime}, z^{\prime}\right)=\left\{\begin{array}{ccc}
1 & z^{\prime} \in\left(z_{i-1}^{\prime}+\Delta_{z}, z^{\prime}{ }_{i+1}-\Delta_{z}\right), & z_{i+1}^{\prime}>z_{i-1}^{\prime} \\
1 & r^{\prime} \in\left(r_{i-1}^{\prime}+\Delta_{r}, r_{i+1}^{\prime}-\Delta_{r}\right), & r_{i+1}^{\prime} \neq r_{i-1}^{\prime} \\
0 & \text { otherwise } &
\end{array}\right. \\
& \Delta_{r}=\left|r_{i+1}^{\prime}-r_{i-1}^{\prime}\right| / 4 \text { and } \Delta_{z}=\left|z^{\prime}{ }_{i+1}-z^{\prime}{ }_{i-1}\right| / 4 .
\end{aligned}
$$

Sub-domains were divided into $N$ sub-intervals and evenly distributed along the sensor core-air interface. Observation points $(r, z)$ were located at the centre of sub-domains (see figure 4) for greatest computational accuracy (Balanis, 1989).

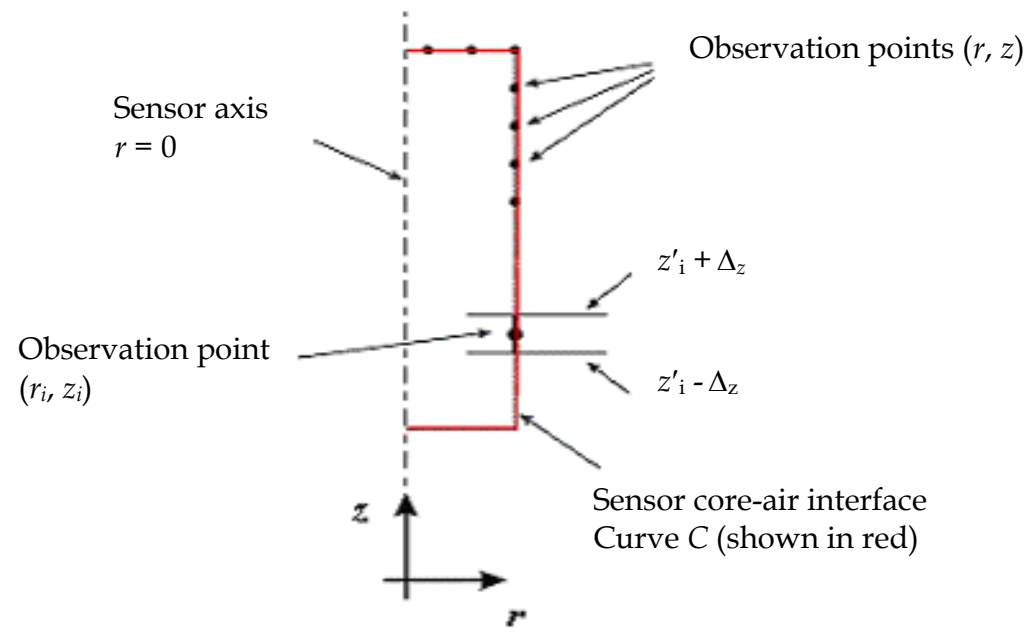

Fig. 4. Discretization of Current $J_{R}$ on the Sensor Core Interface.

\subsection{The total field and internal core field}

When combined the source and scattered field gives the total vector potential $A_{E \varphi}$. For the $n^{\text {th }}$ media layer outside the sensor core: 


$$
\begin{gathered}
A_{E \varphi}(r, z)=\mu_{n} \sum_{i=1}^{N} u_{i} \int_{C} G_{R}^{(n)}\left(r, z ; r^{\prime}, z^{\prime}\right) a_{i}\left(r^{\prime}, z^{\prime}\right) d s^{\prime} \\
\quad+\mu_{n} \iint G_{s}^{(n)}\left(r, z ; r_{0}, z_{0}\right) J_{s \varphi}\left(r_{0}, z_{0}\right) d r_{0} d z_{0}
\end{gathered}
$$

where $J_{s \varphi}$ is the source current distribution:

$$
J_{s \varphi}\left(r_{0}, z_{0}\right)=I N_{c} /\left(l_{2}-l_{1}\right)\left(r_{2}-r_{1}\right)
$$

$\left(l_{2}, l_{1}\right)$ and $\left(r_{2}, r_{1}\right)$ are the length and radial dimensions of the source coil, which is assumed to be rectangular in cross section. Since $A_{I \varphi}$ is solved in exactly the same way as scattered field $A_{R \varphi}$ expand $J_{I \varphi}$ into a similar $N$ term series, letting $b_{i}$ represent the series basis function and $v_{i}$ the expansion coefficient. Given this $b_{i}$ is defined as follows:

$$
b_{i}\left(r^{\prime}, z^{\prime}\right)=\left\{\begin{array}{lcl}
1 & z^{\prime} \in\left(z_{i-1}^{\prime}+\Delta_{z}, z_{i+1}^{\prime}-\Delta_{z}\right), & z_{i+1}^{\prime}>z_{i-1}^{\prime} \\
1 & r^{\prime} \in\left(r_{i-1}^{\prime}+\Delta_{r}, r_{i+1}^{\prime}-\Delta_{r}\right), & r_{i+1}^{\prime} \neq r_{i-1}^{\prime} . \\
0 & \text { otherwise }
\end{array}\right.
$$

Substitution of equation (24) into equation (16) gives:

$$
A_{I \varphi}(r, z)=\mu_{I} \sum_{i=1}^{N} v_{i} \int_{C} G_{I}\left(r, z ; r^{\prime}, z^{\prime}\right) b_{i}\left(r^{\prime}, z^{\prime}\right) d s^{\prime} .
$$

\subsection{Sensor core boundary conditions}

Unknown expansion coefficients $u_{i}$ and $v_{i}$ are determined by applying the sensor core boundary conditions. Since the core is rod shaped, two surfaces exist where boundary conditions must be met. These surfaces are shown below in figure 5 with appropriate unit normal vectors $n$ :

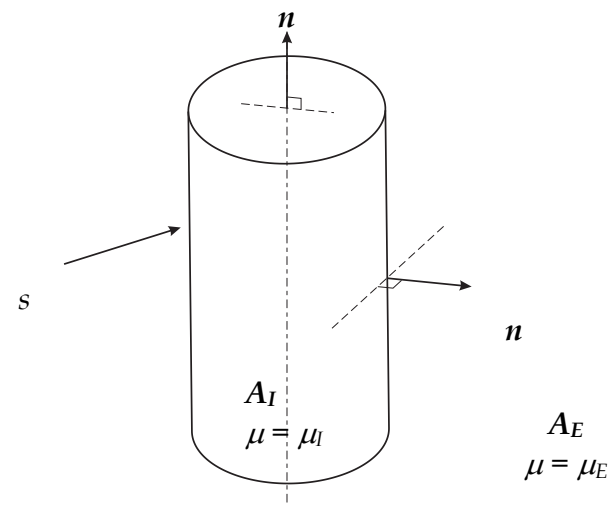

Fig. 5. Core Boundary Unit Normal Vectors $\boldsymbol{n}$. 
If $A_{I}$ is the vector potential inside the core and $A_{E}$ outside, then the boundary conditions for the core-air interface can be shown to be:

$$
\left(1 / \mu_{I}\right) \nabla \times \boldsymbol{A}_{\boldsymbol{I}} \times \boldsymbol{n}=\left(1 / \mu_{E}\right) \nabla \times \boldsymbol{A}_{\boldsymbol{E}} \times \boldsymbol{n}
$$

and

$$
A_{I}=A_{E}
$$

Substituting $\boldsymbol{A}_{\boldsymbol{E}}=\boldsymbol{A}_{\boldsymbol{R}}+\boldsymbol{A}_{\boldsymbol{S}}$ and assuming $\mu_{E}=1$ for free space gives:

$$
\nabla \times \boldsymbol{A}_{\boldsymbol{S}} \times \boldsymbol{n}=\left(\left(1 / \mu_{I}\right) \nabla \times \boldsymbol{A}_{I}-\nabla \times \boldsymbol{A}_{\boldsymbol{R}}\right) \times \boldsymbol{n}
$$

and

$$
A_{S}=A_{I}-A_{R}
$$

Evaluating equation (28) gives the following for the core upper and lower flat faces:

$$
\partial A_{s \varphi}(r, z) / \partial z=\partial\left(A_{I \varphi}(r, z) / \mu_{I}-A_{R \varphi}(r, z)\right) / \partial z
$$

and for the core's central cylindrical face:

$$
(1 / r+\partial / \partial r) A_{s \varphi}(r, z)=(1 / r+\partial / \partial r)\left(A_{I \varphi}(r, z) / \mu_{I}-A_{R \varphi}(r, z)\right)
$$

\subsection{Evaluation of expansion coefficients using the method of weighted residuals}

The two sensor core boundary equations define the relationship between unknown basis function coefficients $u_{i}$ and $v_{i}$. The method of weighted residuals was used to solve these equations, which proceeds by grouping $u_{i}$ and $v_{i}$ together into a single $2 N \times 1$ column matrix $K$ with the following elements:

$$
k_{p}=\left\{\begin{array}{cc}
u_{p} & p=1, \ldots, N \\
v_{p} & p=N+1, \ldots 2 N
\end{array} .\right.
$$

Given $K$ above, define a new $1 \times 2 N$ row matrix $\boldsymbol{\Psi}$ such that:

$$
\boldsymbol{\Psi} \boldsymbol{K}=\left(k_{1} \psi_{1}+\ldots+k_{2 N} \psi_{2 N}\right) .
$$

The following can be seen to apply for element $\psi_{p}$ :

$$
\psi_{p}=\left\{\begin{array}{cc}
\nabla \times\left(\mu_{I}^{-1} \int_{c} G_{I}\left(\boldsymbol{\rho}_{\boldsymbol{q}} ; \boldsymbol{\rho}^{\prime}\right) b_{p}\left(\boldsymbol{\rho}^{\prime}\right) d s^{\prime}-\int_{c} G_{R}^{(n)}\left(\boldsymbol{\rho}_{\boldsymbol{q}} ; \boldsymbol{\rho}^{\prime}\right) a_{p}\left(\boldsymbol{\rho}^{\prime}\right) d s^{\prime}\right) \boldsymbol{a}_{\boldsymbol{\varphi}} \times \boldsymbol{n} & p=1, \ldots, N \\
\int_{c} G_{I}\left(\boldsymbol{\rho}_{\boldsymbol{q}} ; \boldsymbol{\rho}^{\prime}\right) b_{p}\left(\boldsymbol{\rho}^{\prime}\right) d s^{\prime}-\int_{c} G_{R}^{(n)}\left(\boldsymbol{\rho}_{\boldsymbol{q}} ; \boldsymbol{\rho}^{\prime}\right) a_{p}\left(\boldsymbol{\rho}^{\prime}\right) d s^{\prime} & p=N+1, \ldots, 2 N
\end{array}\right.
$$


where $\rho_{\mathrm{q}}$ is a field point at $\left(r_{q}, z_{q}\right), \rho^{\prime}$ is a source point at $\left(r^{\prime}, z^{\prime}\right)$ in sub-domain $p$ and $\boldsymbol{n}$ is a unit normal vector at field point $\left(r_{q}, z_{q}\right)$ on the core boundary surface. Since $2 N$ unknowns in $K$ require the formation of $2 N$ linearly independent equations, define a $2 N \times 1$ column matrix $\boldsymbol{F}$ for the source field at $N$ points $\left\{\left(r_{q}, z_{q}\right) ; q=1, \ldots, N\right\}$ on $C$, with elements:

$$
f_{q}=\left\{\begin{array}{cc}
\nabla \times\left(\iint G_{s}^{(n)}\left(r_{q}, z_{q} ; r_{0}, z_{0}\right) J_{s \phi}\left(r_{0}, z_{0}\right) d r_{0} d z_{0}\right) \boldsymbol{a}_{\varphi} \times \boldsymbol{n} & q=1, \ldots, N \\
\left.\iint G_{s}^{(n)}\left(r_{q}, z_{q} ; r_{0}, z_{0}\right) J_{s \varphi}\left(r_{0}, z_{0}\right) d r_{0} d z_{0}\right) & q=N+1, \ldots, 2 N
\end{array} .\right.
$$

In order to form $2 N$ linearly independent equations, introduce a further $1 \times 2 \mathrm{~N}$ row matrix $\boldsymbol{W}$ and take the inner product $\left\langle\boldsymbol{W}^{\boldsymbol{T}}, \boldsymbol{\Psi}\right\rangle$. Integration is along the entire length of the core-air interface (curve $C$ ) to minimise any residual error, giving:

$$
\left[\int_{c} W^{T} \boldsymbol{\Psi} d s\right] \cdot \boldsymbol{K}=\int_{c} W^{T} \boldsymbol{F} d s
$$

Weight vector $W$ is selected according to one of the following methods (Sadiku, 1992):

- Point collocation.

- Sub-domain collocation.

- Least square.

- Galerkin.

Point collocation was selected because it provided acceptable accuracy for computational effort (Balanis, 1989). Point collocation uses the following weight vector $\boldsymbol{W}$ :

$$
\boldsymbol{W}=\left[\delta\left(c-c_{1}\right), \ldots, \delta\left(c-c_{2 N}\right)\right]
$$

Collocation points on $C$ are chosen to coincide with basis function observation points, where the following applies for weight element $w_{q}$ :

$$
\delta\left(c-c_{q}\right)=\left\{\begin{array}{lc}
\infty & \text { if } \quad\left(c=c_{q}\right) \\
0 & \text { otherwise }
\end{array}\right.
$$

Recognising that $\int_{x_{i}^{-}}^{x_{i}^{+}} \delta\left(x-x_{i}\right) d x=1$ and inserting this into row $q$ of equation (36), gives:

$$
f_{q} \int_{C_{q}^{-}}^{C_{q}^{+}} \delta\left(c-c_{q}\right) d s=\psi_{1} u_{1} \int_{C_{q}^{-}}^{C_{q}^{+}} \delta\left(c-c_{q}\right) d s+\cdots+\psi_{2 N} v_{2 N} \int_{C_{q}^{-}}^{C_{q}^{+}} \delta\left(c-c_{q}\right) d s
$$

Evaluating equation (39) for all $N$ collocation points leads to the matrix equation for $u_{i}$ and $v_{i}$ : 


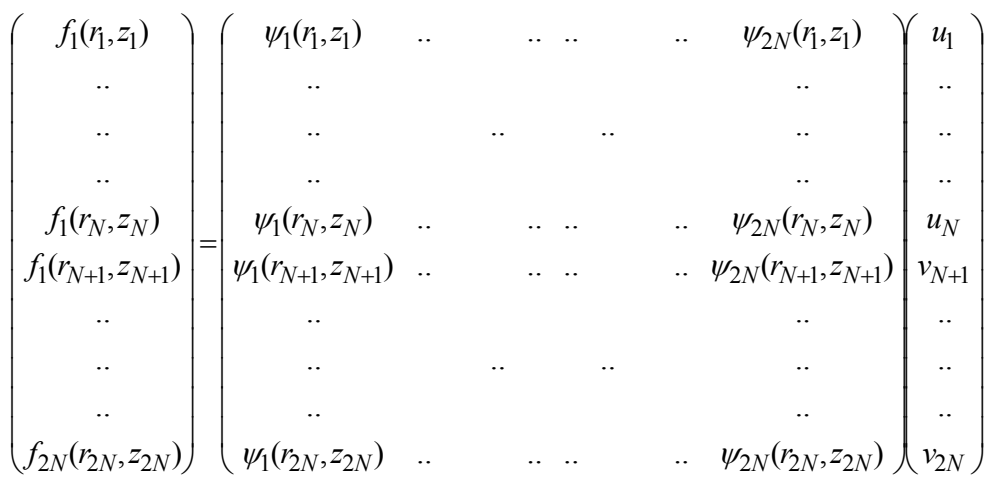

\section{Numerical model implementation}

In previous section, a set of partial differential equations were developed to model the vector potential fields present in the regions bounding a ferrite-cored eddy current sensor; sensor regions were considered to be source-less with imaginary surface currents imposed at region interfaces. Green's functions were determined for all bounded regions. A novel set of Basis functions were introduced to reproduce the surface currents present on the sensor core-air interface, which were then formed into a system of $2 N$ linearly independent equations. A matrix method was finally developed to solve these equations using the method of weighted residuals.

The matrix method was further developed in this section in order to calculate sensor coil impedance and induced voltage. An efficient material profile function $m(\alpha)$ for modelling the interaction between the sensor and test material was also developed and verified. A novel form of parameterisation is adopted for $m(\alpha)$. The accuracy and convergence of vector potential fields generated is reviewed and a new free-space Green's function introduced.

\subsection{The material profile function $m(\alpha)$ for stratified layers}

Section 2.1 introduced the medium as being comprised of M planar layers. Each layer had medium properties that were considered to be isotropic, homogeneous and linear. Any change between the electrical and magnetic properties of the layers was a step change. This approach enabled Cheng and co-workers to successfully model non-homogeneous materials using piecewise constant approximations (Cheng et al, 1971). Such non-linear material profiles might be produced, as an example, by coating a substrate, by case hardening, heat treatment, ion bombardment, or by chemical processing. A recently developed alternative method used hyperbolic tangential profiles to represent near surface changes in conductivity (Uzal et al, 1993). The method proposed by Cheng was adopted here due to it being more flexible. Applying boundary equations (26) and (27) to Green's function (12) for the $n^{\text {th }}$ media layer below the coil gives:

$$
B_{n-1}=\frac{1}{2} e^{\alpha_{-1} z_{n}}\left[\left(1+\beta_{n-1, n}\right) e^{-\alpha_{n} z_{n}} B_{n}+\left(1-\beta_{n-1, n}\right) e^{\alpha_{n} z_{n}} C_{n}\right]
$$


and

$$
C_{n-1}=\frac{1}{2} e^{\alpha_{n-1} z_{n}}\left[\left(1-\beta_{n-1, n}\right) e^{-\alpha_{n} z_{n}} B_{n}+\left(1+\beta_{n-1, n}\right) e^{\alpha_{n} z_{n}} C_{n}\right]
$$

where:

$$
\beta_{n-1, n}=\beta_{n} / \beta_{n-1}
$$

and

$$
\beta_{n}=\alpha_{n} / \mu_{n}
$$

Taking the coil region (regions 1 and 2) to have the properties $\alpha_{0}, \beta_{0}$ and $\mu_{0}$, and giving consideration to the presence of a source give:

$$
-B_{2} e^{-\alpha_{0} z_{0}}+C_{2} e^{\alpha_{0} z_{0}}=-B_{1} e^{-\alpha_{0} z_{0}}+C_{1} e^{\alpha_{0} z_{0}}+\frac{\alpha}{\beta_{0}} r_{0} J_{1}\left(\alpha r_{0}\right) .
$$

and

$$
-B_{2} e^{-\alpha_{0} z_{0}}+C_{2} e^{\alpha_{0} z_{0}}=-B_{1} e^{-\alpha_{0} z_{0}}+C_{1} e^{\alpha_{0} z_{0}}
$$

Cheng and co workers showed that unknown coefficients $B_{n}$ and $C_{n}$ were dramatically simplified using a matrix method (Cheng et al, 1971). This method defined the following matrices, a $2 \times 1$ coefficient column matrix:

$$
\boldsymbol{A}_{n}=\left[\begin{array}{l}
B_{n} \\
C_{n}
\end{array}\right]
$$

and a $2 \times 2$ transformation matrix $T_{n-1, n}$, with the elements:

$$
\begin{gathered}
\left(\boldsymbol{T}_{\boldsymbol{n}-1, \boldsymbol{n}}\right)_{11}=\frac{1}{2}\left(1+\beta_{n-1, n}\right) e^{\left(\alpha_{n-1}-\alpha_{n}\right) z_{n}} \\
\left(\boldsymbol{T}_{\boldsymbol{n}-1, \boldsymbol{n}}\right)_{12}=\frac{1}{2}\left(1-\beta_{n-1, n}\right) e^{\left(\alpha_{n-1}+\alpha_{n}\right) z_{n}} \\
\left(\boldsymbol{T}_{\boldsymbol{n}-1, \boldsymbol{n}}\right)_{21}=\frac{1}{2}\left(1-\beta_{n-1, n}\right) e^{-\left(\alpha_{n-1}+\alpha_{n}\right) z_{n}} \\
\left(\boldsymbol{T}_{\boldsymbol{n}-1, \boldsymbol{n}}\right)_{22}=\frac{1}{2}\left(1+\beta_{n-1, n}\right) e^{-\left(\alpha_{n-1}-\alpha_{n}\right) z_{n}}
\end{gathered}
$$

Successive multiplication of $\boldsymbol{T}_{n-1, n}$ gives the following:

$$
A_{2}=\left[\begin{array}{l}
B_{2} \\
C_{2}
\end{array}\right]=T_{2,3} \cdot T_{3,4} \ldots T_{M-2, M-1} \cdot T_{M-1, M} \cdot A_{M}
$$


where

$$
\boldsymbol{A}_{\boldsymbol{M}}=\left[\begin{array}{c}
0 \\
C_{M}
\end{array}\right]
$$

If $\boldsymbol{V}(n, M)$ is a $2 \times 2$ matrix, then define:

$$
\boldsymbol{V}(n, M)=\boldsymbol{T}_{2,3} \cdot \boldsymbol{T}_{3,4} \cdots \boldsymbol{T}_{M-2, M-1} \cdot \boldsymbol{T}_{M-1, M}
$$

Evaluating the above gives the Green's functions for the regions bounding the coil:

Region 1

$$
G_{S}^{(1)}\left(r, z, r_{0}, z_{0}\right)=\int_{0}^{\infty} \alpha r_{0} J_{1}\left(\alpha r_{0}\right) J_{1}(\alpha r)\left(\frac{v_{12}(2, M)}{v_{22}(2, M)} \cdot e^{-\alpha_{0} z_{0}}+e^{\alpha_{0} z_{0}}\right) \cdot e^{-\alpha_{0} z} d \alpha
$$

Region 2

$$
G_{S}^{(2)}\left(r, z, r_{0}, z_{0}\right)=\int_{0}^{\infty} \alpha r_{0} J_{1}\left(\alpha r_{0}\right) J_{1}(\alpha r)\left(\frac{v_{12}(2, M)}{v_{22}(2, M)} \cdot e^{-\alpha_{0} z}+e^{\alpha_{0} z}\right) \cdot e^{-\alpha_{0} z_{0}} d \alpha
$$

A new coil region (region 12) can also be defined for coils with finite length and radial dimensions by adding vector potential $A^{(1)}$ and $A^{(2)}$ and applying relevant boundary conditions on coil length Z (Dodd \& Deeds, 1968).

From the above, the following relationship is evident:

$$
m=\frac{v_{12}(2, M)}{v_{22}(2, M)}
$$

Material profile function $m$ is dependent only on the media properties and is independent of coil geometry and coil lift-off; $m$ not only applies to the source field $A_{S}$, but also to the scattered field $A_{R}$, which makes this function a universal profile function. If the material under test is comprised of two layers for simplicity, a conductive coating of thickness $Z_{c}$ (region 3) deposited on a magnetic substrate (region 4), then the material profile function $m$ can be shown to be (Dodd \& Deeds, 1968):

$$
m(\alpha)=\frac{\left(\alpha+\alpha_{3}\right)\left(\alpha_{3}-\alpha_{4} / \mu_{4}\right)+\left(\alpha-\alpha_{3}\right)\left(\alpha_{3}+\alpha_{4} / \mu_{4}\right) \cdot e^{2 \cdot Z_{c} \cdot \alpha_{3}}}{\left(\alpha-\alpha_{3}\right)\left(\alpha_{3}-\alpha_{4} / \mu_{4}\right)+\left(\alpha+\alpha_{3}\right)\left(\alpha_{3}+\alpha_{4} / \mu_{4}\right) \cdot e^{2 \cdot Z_{c} \cdot \alpha_{3}}}
$$

where

$$
\alpha_{3}=\sqrt{\alpha^{2}+j \omega \mu_{3} \sigma_{3}} \text { and } \alpha_{4}=\sqrt{\alpha^{2}+j \omega \mu_{4} \sigma_{4}} \text {. }
$$




\subsection{Implementation of the material profile function}

The material profile function $m$ is actually a function of many variables. Most of these variables however can be regarded as constant for a given test, making the material profile function a function of spatial frequency a only. For a large number of medium layers, at least 40 to accurately represent continuously varying profiles (Uzal et al, 1993), the evaluation of $V_{12}$ and $V_{22}$ begins to become computationally prohibitive. Not only does the amount of matrix algebra required to calculate $m(\alpha)$ dramatically increase, but this calculation must be repeated for every element of matrix equation (40). A more efficient approach replaces $m(\alpha)$ in its matrix form with a spline curve. The oscillatory nature of high degree polynomial approximations, such as least squares regression, discounts their use. In order to assess the suitability of cubic spline interpolation it is necessary to determine the general form and amount of variation expected for $m(\alpha)$. Given this, consider a two layer medium defined by equation (58), where angular frequency $\omega$ ranges from $\omega=2 \pi \cdot 100 \mathrm{rads} / \mathrm{sec}$ to $\omega=2 \pi \cdot 3 \cdot 10^{4} \mathrm{rads} / \mathrm{sec}$ and coating thickness $Z_{c}$ (region 3) ranges from $Z_{c}=0 \mu \mathrm{m}$ to $300 \mu \mathrm{m}$. A worse case of copper plating on steel is assumed. The following two graphs show real and imaginary components of $m(\alpha)$ for these conditions.

Examination of figure $6 \mathrm{a}$ and $6 \mathrm{~b}$ clearly shows that $m(\alpha)$ has considerable variation below $\alpha$ $=10^{4}$, but that above this it is relatively smooth. Assuming that $m(\alpha)$ is defined on the interval $\alpha \in\{a, b\}$ and that a clamped boundary is used, let cubic polynomial $S_{\mathrm{j}}$ occur on subinterval $\left[\alpha_{j}, \alpha_{j+1}\right]$. Given this it can be shown that the maximum error occurs when: (Burden \& Faires, 1989):

$$
\max _{a \leq \alpha \leq b}|m(\alpha)-S(\alpha)| \leq \frac{5 \cdot M_{\alpha}}{384} 0 \leq j \leq n-1{ }^{\max }\left(\alpha_{j+1}-\alpha_{j}\right)^{4}
$$

where

$$
M_{\alpha} \geq \max _{a \leq \alpha \leq b}\left|m^{4}(\alpha)\right| \text { and } a=\alpha_{0}\left\langle\alpha _ { 1 } \left\langle\ldots \left\langle\alpha_{n}=b\right.\right.\right.
$$

From above, interpolation error can be linked with the maximum subinterval step size $\max \left[\alpha_{j}, \alpha_{j+1}\right]$ and the maximum $4^{\text {th }}$ derivative of $m(\alpha)$. Since the maximum derivative error is always below $\alpha=2 \cdot 10^{3}$ and since $m(\alpha)$ is almost linear above $\alpha=10^{4}$, it seems reasonable to reduce subinterval step size for low $\alpha$ and increase it above $\alpha=10^{4}$. This adjustment enables the interpolating cubic polynomials $S_{j}$ to more accurately reproduce data in regions of maximum variation, whilst minimising the total number of subinterval domains. Empirical study showed that the optimum choice for $\alpha_{j}$ is:

$$
\alpha_{j}=0.035 \cdot\left((j+1)^{3}+j^{3}\right)
$$

where

$$
j \in\{0,1, \ldots, 111\} .
$$




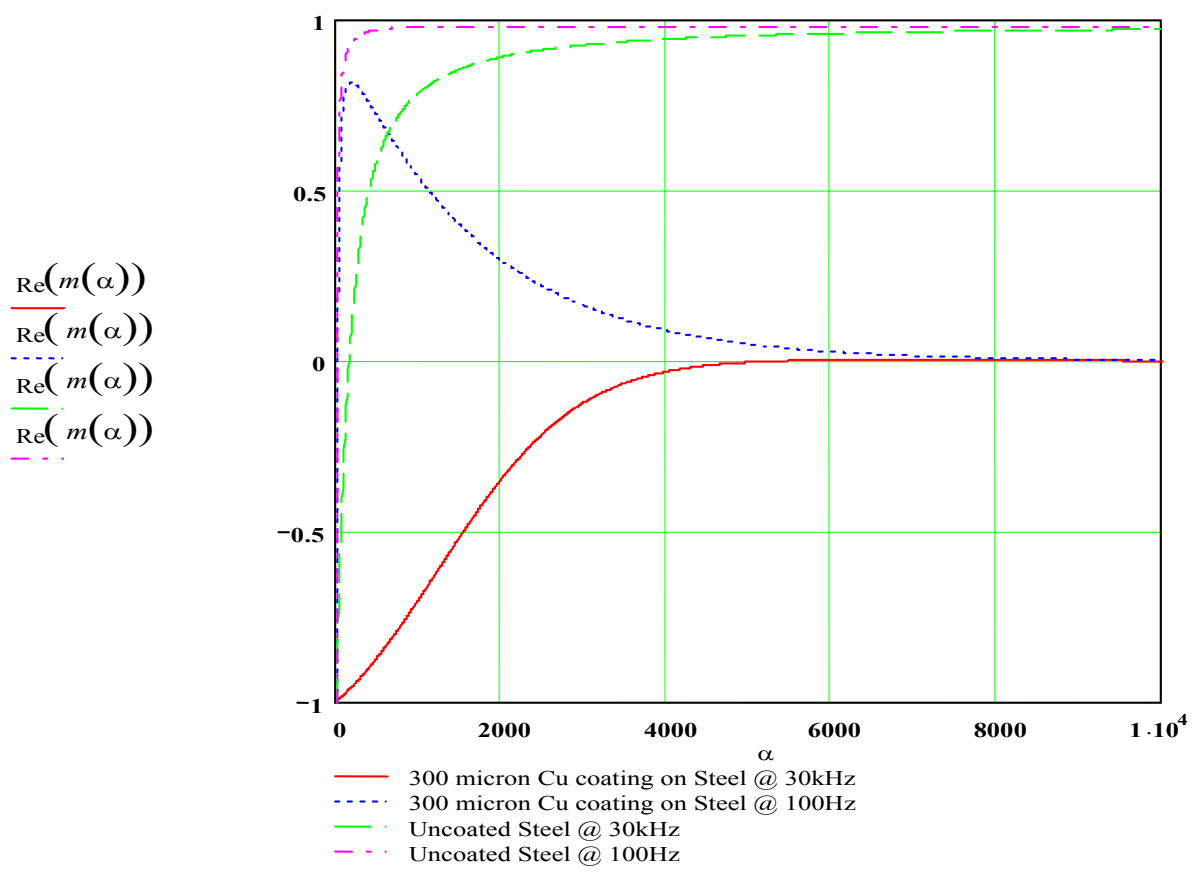

Fig. 6a. Real Component of Material Profile Function $m(\alpha)$.
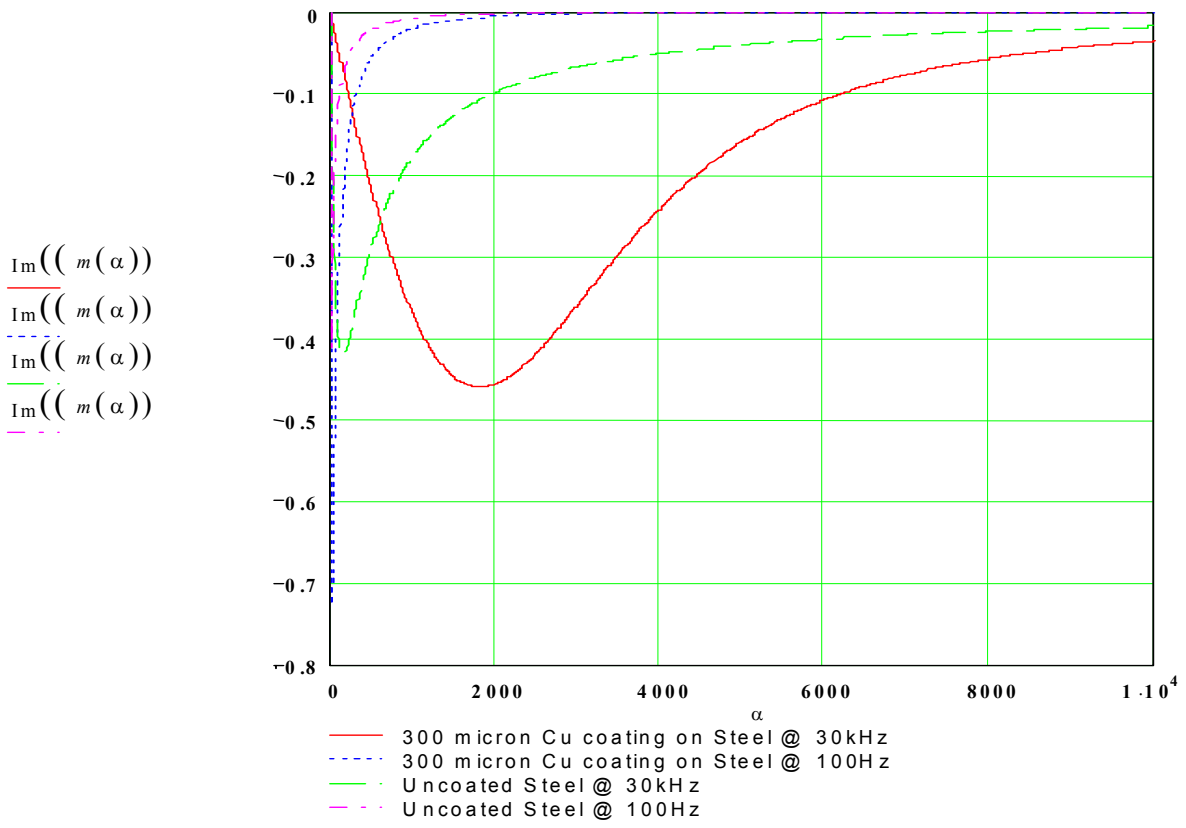

Fig. 6b. Imaginary Component of Material Profile Function $m(\alpha)$. 


\subsection{Material profile function testing and evaluation}

A typical spline curve is given in figure 7 for a two layer material: substrate (region $4: \mu_{4}=$ $100, \sigma_{4}=10 \mathrm{MS} / \mathrm{m}$ ) and coating (region 3: $\mu_{3}=1, \sigma_{3}=58 \mathrm{MS} / \mathrm{m}$, coating thickness $Z_{c}=300$ $\mu \mathrm{m})$ for an excitation frequency $\omega=2 \pi \cdot 30 \cdot 10^{3} \mathrm{rads} / \mathrm{sec}$.

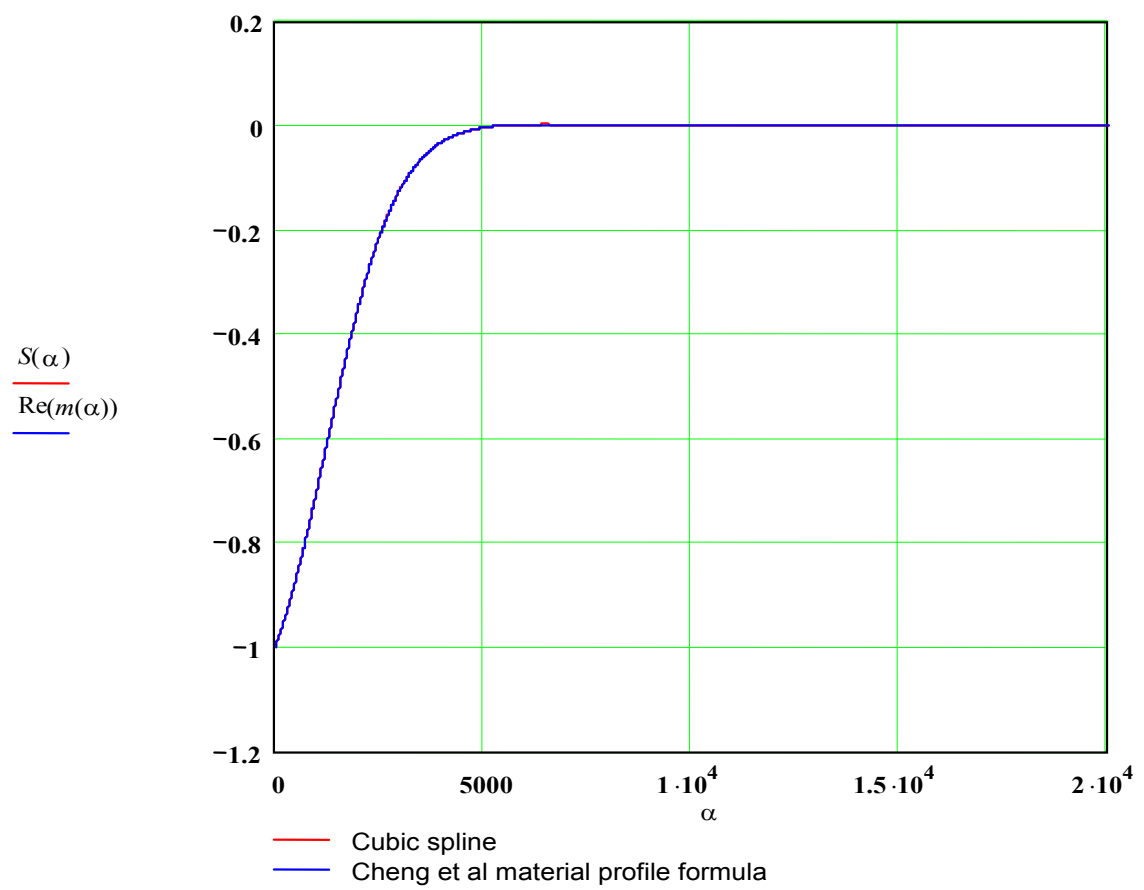

Fig. 7. Spline Approximation $S(\alpha)$ of Cheng Formula $\operatorname{Re}(m(\alpha))$.

The major benefit of this approach is that the cubic polynomial coefficients for all $S_{j}$ need only be calculated once, making the method very rapid.

\subsection{The convergence of the source coil fields}

The vector potentials required for evaluating the regions bounding the source coil are derived from equations (11) and (12), which are improper integrals. Since an explicit antiderivative does not exist for these equations, numerical quadrature was be used. The convergence of these integrals can be studied by considering the form of their integrand for large $\alpha$, which can be represented in the following way:

$$
\int_{a}^{\xi} \frac{d \alpha}{\alpha^{n}}=\frac{\xi^{1-n}-a^{1-n}}{1-n}
$$


If $n>1$ then $\xi^{1-n} \rightarrow 0$ for $\xi \rightarrow \infty$. Given this equation (61) is convergent. Stephenson generalises this further by redefining equation (61) as (Stephenson, G. 1974):

$$
\int_{a}^{\zeta} \frac{g(\alpha)}{\alpha^{n}}
$$

where $g(\alpha)$ is some arbitrary function that is bounded and non-zero.

In this instance (62) is said to be convergent if $n>1$. Given this, it seems reasonable to assume that a large positive value for $n$ is required for a high rate of convergence. An example of this is given for the self inductance $L$ of an air-cored coil (Dodd \& Deeds, 1968):

$$
L=\pi n_{C}^{2} \mu \int_{0}^{\infty} \frac{1}{\alpha^{5}} I\left(r_{2}, r_{1}\right)^{2}\left\{2 \alpha\left(l_{2}-l_{1}\right)+2 e^{-\alpha\left(l_{2}-l_{1}\right)}-2+\left(e^{-2 \alpha l_{2}}+e^{-2 \alpha l_{1}}-2 e^{-\alpha\left(l_{2}-l_{1}\right)}\right) m\right\} d \alpha
$$

The function shown above has a very rapid rate of convergence due to $\alpha$ being raised to the $5^{\text {th }}$ power. Application of the sensor core boundary equation (28) leads to five core equations for the source coil magnetic flux density $\boldsymbol{B}_{S}$, which are given below:

$$
\begin{gathered}
B_{S z}^{(1)}=\frac{1}{2} n_{c} I \mu \int_{0}^{\infty} \frac{1}{\alpha^{2}} I\left(r_{2}, r_{1}\right) J_{0}(\alpha r) e^{-\alpha z}\left\{e^{\alpha l_{2}}-e^{\alpha l_{1}}-\left(e^{-\alpha l_{2}}-e^{-\alpha l_{1}}\right) m(\alpha)\right\} d \alpha \\
B_{S z}^{(2)}=\frac{1}{2} n_{c} I \mu \int_{0}^{\infty} \frac{1}{\alpha^{2}} I\left(r_{2}, r_{1}\right) J_{0}(\alpha r)\left(e^{-\alpha l_{1}}-e^{-\alpha l_{2}}\right)\left(e^{\alpha z}-m(\alpha) e^{-\alpha z}\right) d \alpha \\
B_{S z}^{(12)}=\frac{1}{2} n_{c} I \mu \int_{0}^{\infty} \frac{1}{\alpha^{2}} I\left(r_{2}, r_{1}\right) J_{0}(\alpha r)\left\{2-e^{\alpha\left(z-l_{2}\right)}-e^{-\alpha\left(z-l_{1}\right)}+e^{-\alpha z}\left(e^{-\alpha l_{1}}-e^{-\alpha l_{2}}\right) m(\alpha)\right\} d \alpha \\
B_{S r}^{(1)}=-\frac{1}{2} n_{c} I \mu \int_{0}^{\infty} \frac{1}{\alpha^{2}} I\left(r_{2}, r_{1}\right) J_{1}(\alpha r) e^{-\alpha z}\left\{e^{\alpha l_{2}}-e^{\alpha l_{1}}-\left(e^{-\alpha l_{2}}-e^{-\alpha l_{1}}\right) m(\alpha)\right\} d \alpha \\
B_{S r}^{(2)}=-\frac{1}{2} n_{c} I \mu \int_{0}^{\infty} \frac{1}{\alpha^{2}} I\left(r_{2}, r_{1}\right) J_{1}(\alpha r)\left(e^{-\alpha l_{1}}-e^{-\alpha l_{2}}\right)\left(e^{\alpha z}-m(\alpha) e^{-\alpha z}\right) d \alpha
\end{gathered}
$$

Boundary equations (64) - (68) have a rate of convergence no worse than $\alpha^{-2}$. Comparison with that of the source coil inductance indicates that the rate of convergence of source coil field vectors is relatively poor. 


\subsection{Convergence of the basis function fields}

The field generated by an $i^{\text {th }}$ basis function located on the cylindrical face of the sensor core is given by:

$$
A_{R \varphi}^{*}(r, z)=\mu u_{i} \int_{z_{i}-\Delta z}^{z_{i}+\Delta z} G_{R}^{(n)}\left(r, z ; r^{\prime}, z^{\prime}\right) d z^{\prime}
$$

where the total scattered field $A_{R \varphi}(r, z) \cong \sum_{i=1}^{N} A_{R \varphi}^{*}(r, z)$ and $\Delta_{z}=\left|z_{i+1}-z_{i-1}\right| / 4$.

If the axial coordinates of the basis function are $l_{a 2}=z_{i}+\Delta z$ and $l_{a 1}=z_{i}-\Delta z$, with radial coordinate $r_{a}$, equation (69) becomes:

$$
A_{R \varphi}^{*}=\frac{r_{a}}{2} \mu u_{i} \int_{0}^{\infty} \frac{1}{\alpha} J_{1}\left(\alpha r_{a}\right) J_{1}(\alpha r)\left\{2-e^{\alpha\left(z-l_{a 2}\right)}-e^{-\alpha\left(z-l_{a 1}\right)}+e^{-\alpha z}\left(e^{-\alpha l_{a 1}}-e^{-\alpha l_{a 2}}\right) m(\alpha)\right\} d \alpha
$$

Which assumes that field point $(r, z)$ is bounded, with $l_{a 2} \leq z \leq l_{a 1}$.

The components of flux density $\boldsymbol{B}_{\boldsymbol{R}}^{*}=B_{R r}^{*} \boldsymbol{a}_{\boldsymbol{r}}+B_{R z}^{*} \boldsymbol{a}_{z}$ for equation (70) are:

$$
\begin{aligned}
& B_{R r}^{*}=\frac{r_{a}}{2} \mu u_{i} \int_{0}^{\infty} J_{1}\left(\alpha r_{a}\right) J_{1}(\alpha r)\left\{-e^{\alpha\left(z-l_{a 2}\right)}-e^{-\alpha\left(z-l_{a 1}\right)}-e^{-\alpha z}\left(e^{-\alpha l_{a 1}}-e^{-\alpha l_{a 2}}\right) m(\alpha)\right\} d \alpha \\
& B_{R z}^{*}=\frac{r_{a}}{2} \mu m_{i} \int_{0}^{\infty} J_{1}\left(\alpha r_{a}\right) J_{0}(\alpha r)\left\{2-e^{\alpha\left(z-l_{a 2}\right)}-e^{-\alpha\left(z-l_{a 1}\right)}+e^{-\alpha z}\left(e^{-\alpha l_{a 1}}-e^{-\alpha l_{a 2}}\right) m(\alpha)\right\} d \alpha
\end{aligned}
$$

It is clear that a basis function vector potential has a rate of convergence that is poor, the convergence of it's flux density vector $\boldsymbol{B}_{\boldsymbol{R}}^{*}$ is even worse. A significant benefit in terms of computational efficiency and accuracy is gained if a method can be found to improve the convergence of these field equations. Note that the fields above and below the basis function, as well as the basis functions on the end faces of the core, have been omitted for brevity.

\subsection{The modified free space green's function $G_{0}\left(r, z ; r^{\prime}, z^{\prime}\right)$}

It is evident that field equations have a poor rate of convergence. Considering only the basis function fields, separate equation (72) into two parts, which are given on the following: 


$$
\begin{aligned}
& B_{R z}^{*}=\frac{1}{2} \mu u_{i} \int_{0}^{\infty} J_{1}\left(\alpha r_{a}\right) J_{0}(\alpha r) e^{-\alpha z}\left\{e^{\alpha l_{a 2}}-e^{\alpha l_{a 1}}\right\} d \alpha+ \\
& \frac{1}{2} \mu u_{i} \int_{0}^{\infty} J_{1}\left(\alpha r_{a}\right) J_{0}(\alpha r) e^{-\alpha z}\left(e^{-\alpha l_{a 1}}-e^{-\alpha l_{a 2}}\right) m(\alpha) d \alpha
\end{aligned}
$$

Assuming that all other field equations can be treated in the same way, a comparison of the integrands of equation (73) is shown in a normalised form in figure 8, with $m(\alpha)=1.0$.

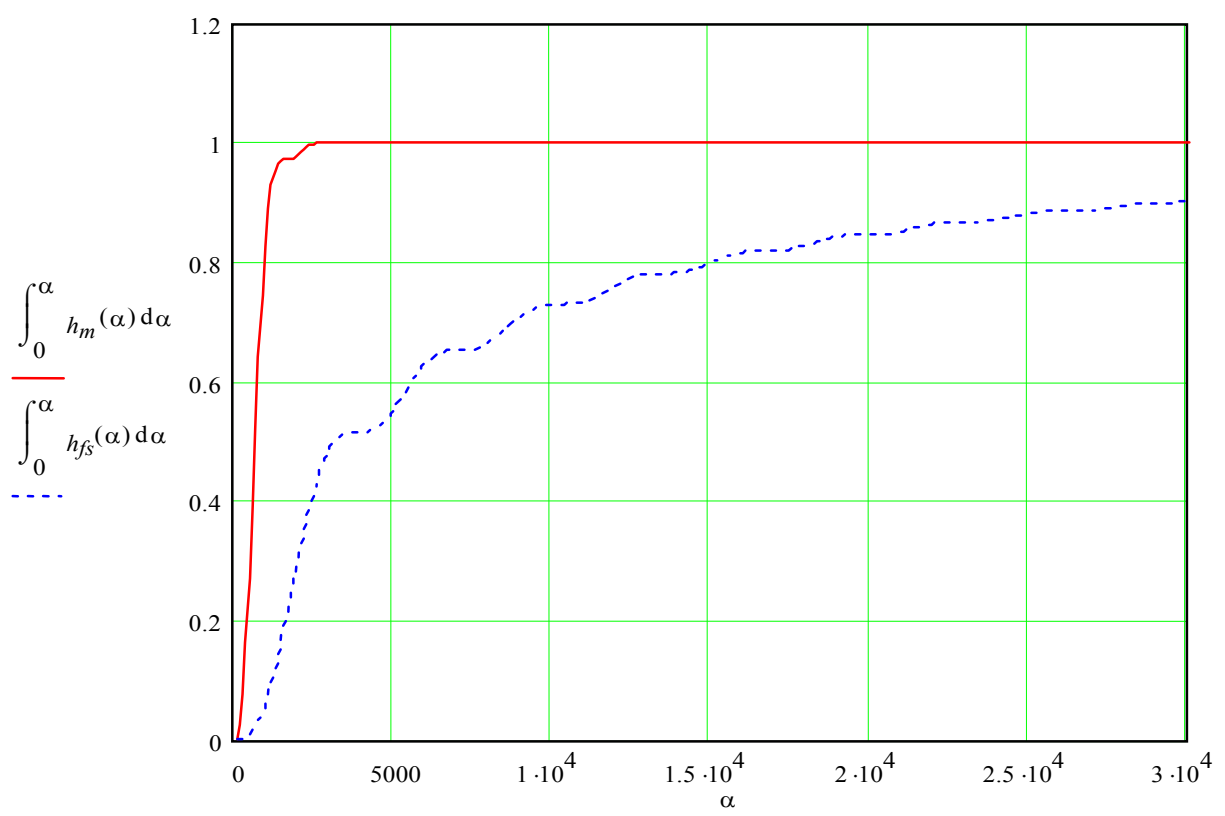

Fig. 8. Free-Space $h_{f s}(\alpha)$ and Material Dependent $h_{m}(\alpha)$ Convergence.

It is clear from Figure 8 that separating the field equations into two terms, and considering only the material dependent term $h_{m}(\alpha)$ gives a function with a very rapid rate of convergence. An equation replacing the free-space or material independent term $h_{f_{s}}(\alpha)$ now needs to be determined. Let the delta function coil representing the material independent Green's function $G_{0}\left(r, z ; r^{\prime}, z^{\prime}\right)$ be formed from discrete current elements $I d S$. See figure 9. 


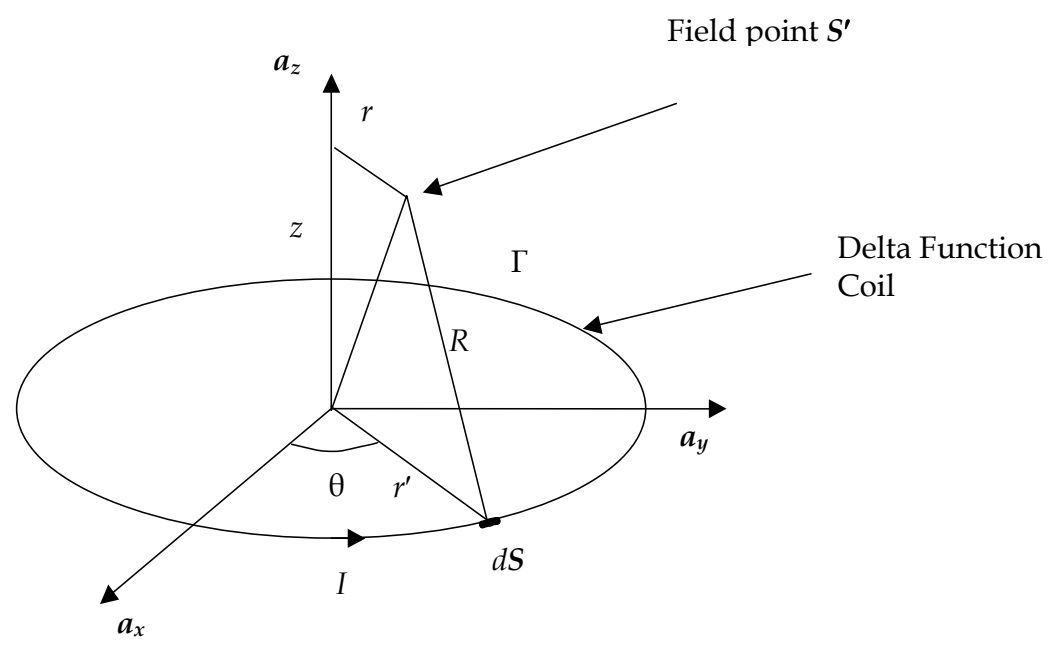

Fig. 9. Current Element IdS forming a Delta Function Coil.

The magnetic vector potential generated by $I d S$ is given as:

$$
\boldsymbol{A}_{\boldsymbol{R}}^{*}=\mu I \int_{\Gamma} \frac{d \boldsymbol{S}}{R}
$$

where $\mu$ is media permeability and $I$ is current.

If $\boldsymbol{S}=r^{\prime}\left(\cos (\theta) \boldsymbol{a}_{\boldsymbol{x}}+\sin (\theta) \boldsymbol{a}_{\boldsymbol{y}}\right), \quad \boldsymbol{S}^{\prime}=r \boldsymbol{a}_{\boldsymbol{x}}+z \boldsymbol{a}_{z}$ and $R=\left|\boldsymbol{S}^{\prime}-\boldsymbol{S}\right|$, then the free space Green's function $G_{0}\left(r, z ; r^{\prime}, z^{\prime}\right)$ is of the following form:

$$
G_{0}\left(r, z ; r^{\prime}, z^{\prime}\right)=\frac{I}{4 \pi} \int_{0}^{\pi} \frac{\mu \cdot r^{\prime} \cdot \cos (\theta) d \theta}{\sqrt{\left(r-r^{\prime} \cdot \cos (\theta)\right)^{2}+\left(z-z^{\prime}\right)^{2}+\left(r^{\prime} \cdot \sin (\theta)\right)^{2}}}
$$

Integration of $G_{0}\left(r, z ; r^{\prime}, z^{\prime}\right)$ over $r$ or $z$ gives the relevant equation for the basis functions.

Equation (75) satisfies all the requirements of the Green's function (Sadiku, 1992). Highly accurate calculations of field quantities were found to be possible using this equation.

\section{Testing and evaluation}

Nearly all eddy current investigations are conducted in the sensor coil region by determining coil impedance. Given this, if the source coil is densely and uniformly wound with a rectangular cross section, having the radial and axial dimensions $\left(r_{2}, r_{1}\right)$ and $\left(l_{2}, l_{1}\right)$, the induced voltage $V$ across the coil will be equal to:

$$
V=\frac{j \omega 2 \pi N_{c}}{\left(l_{2}-l_{1}\right)\left(r_{2}-r_{1}\right)} \int_{l_{1}}^{l_{2}} \int_{r_{1}}^{r_{2}} r\left(A_{S \varphi}^{(12)}(r, z)+A_{r \varphi}(r, z)\right) d r d z,
$$


where $N_{c}$ is the number of turns on the source coil. Coil impedance $Z$ can be found by simply dividing $V$ by source current $I$.

Evaluation of the sensor model proceeded by defining the dimensional and physical properties of the sensor of section 2, which are shown below in table 1 . Note that no information for the two pickup coils is given as this is the subject of future work.

\begin{tabular}{|c|c|c|c|}
\hline \multicolumn{2}{|c|}{ Sensor Core } & \multicolumn{2}{c|}{ Sensor Source Coil } \\
\hline Sensor Lift-off: & $0.50 \mathrm{~mm}$ & Source Coil $r_{1}:$ & $1.45 \mathrm{~mm}$ \\
\hline Core Radius: & $0.99 \mathrm{~mm}$ & Source Coil $r_{2}:$ & $3.175 \mathrm{~mm}$ \\
\hline Core Length: & $6 \mathrm{~mm}$ & Source Coil $l_{1}:$ & $3.005 \mathrm{~mm}$ \\
\hline Core Permeability & 1000 & Source Coil $l_{2}:$ & $3.845 \mathrm{~mm}$ \\
\hline & & Source Coil Turns $N_{c}:$ & 294 \\
\hline
\end{tabular}

Table 1. Sensor Properties.

Matrix equation (40) was solved for coefficients $u_{i}$ and $v_{i}$ using Mathcad, version 11.0a and source coil impedance determined from equation (76). It was found empirically that 80 collocation points spread evenly along the core-air interface $C$ provided good results.

Source coil self inductance $L$ and resistance $R$ was calculated for differing sensor lift off over steel with the following properties: relative permeability $\mu_{r}=95.6$ and conductivity $\sigma=$ $8.4 \times 10^{6} \mathrm{~S} / \mathrm{m}$. As a comparison, the sensor model of table 1 was also simulated using the commercial FEM solver MagNet, version 6.25. The results of this, displayed in the form of a normalised impedance plane diagram, are shown in figure 10.

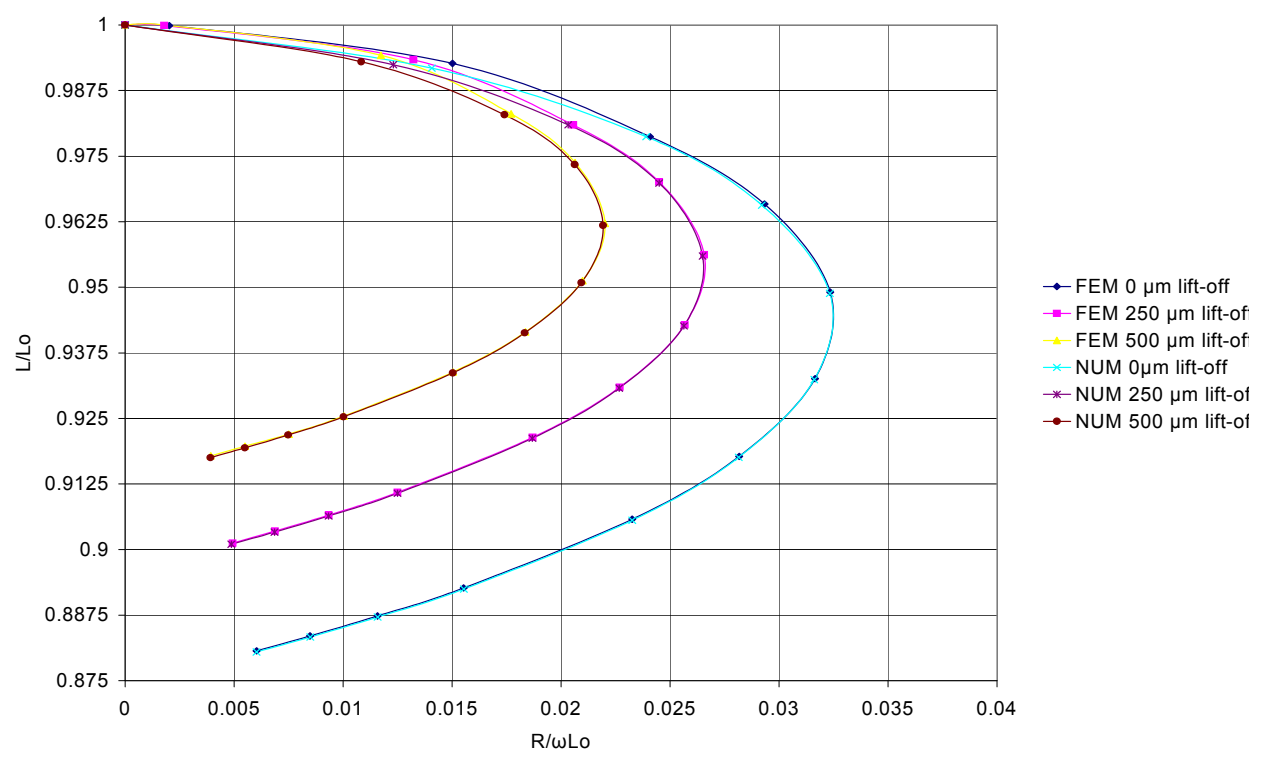

Fig. 10. Normalised Impedance Plane Diagram of Sensor. 
Changes to the value of core permeability $\mu_{\mathrm{r}}$ were also simulated for the sensor held in free space, positioned above a solid copper substrate $(f=30 \mathrm{kHz})$ and finally above 100 $\mu \mathrm{m}$ of copper plating on steel $(f=10 \mathrm{kHz})$. The results of this simulation are displayed in figure 11 .

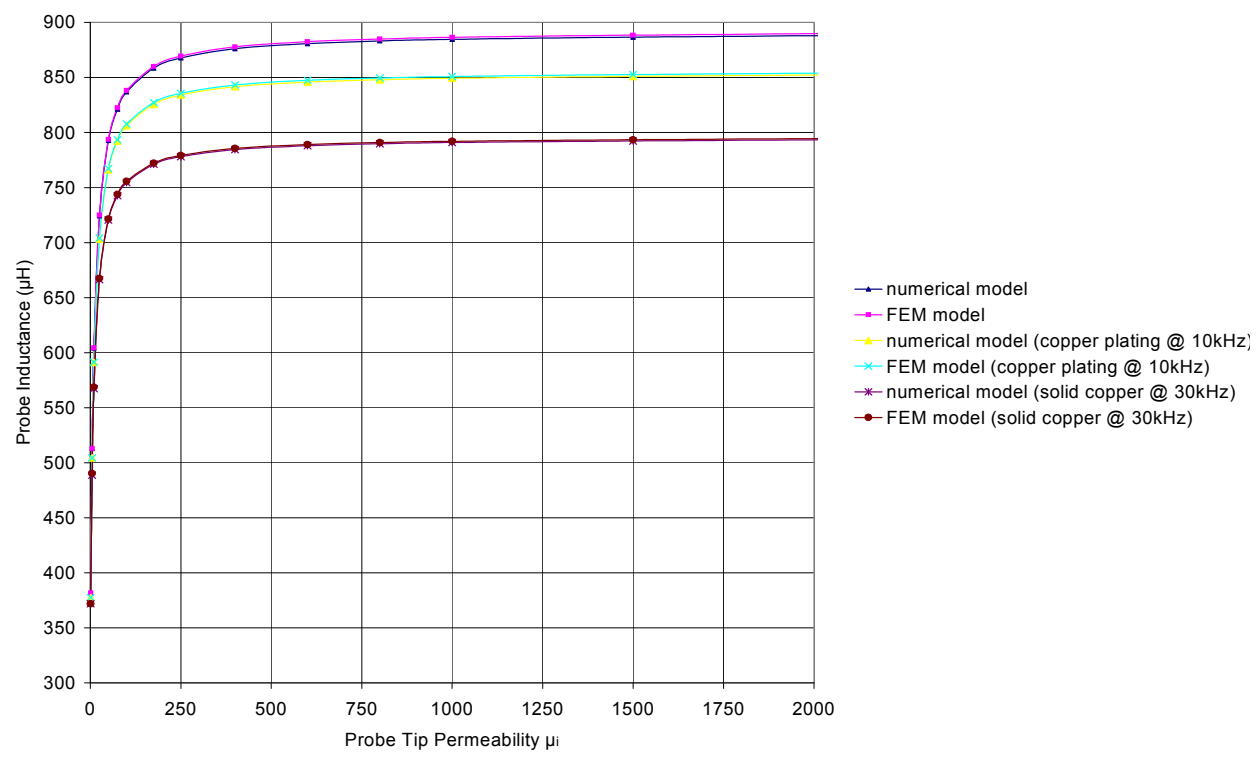

Fig. 11. Source Coil Inductance as a Function of Core Permeability.

\section{Conclusion}

The benefits of the magnetic moment method developed in this work are:

- Only points on the closed surface bounding the sensor core are discretised.

- The use of a spline function to replace the material dependent matrices $V(M, 1)_{12} / V(M, 1)_{22}$ of Cheng, Dodd and Deeds (Equation 7), allows for a potentially infinite number of stratified layers to be used to represent non-linear material profiles, with no penalty in terms of computation time or accuracy.

- Separating the basis functions into free-space static and substrate dependent dynamic terms, allows for more efficient computation of magnetic fields on the equivalent boundary surface. Static field components (free space components) only need to be computed once for any given simulation session.

- For a given lift-off and material profile function $m(\alpha)$, probe tip permeability $\mu_{\mathrm{i}}$ can be varied without the need to recalculate basis function fields. 
Future work entails implementing a Galerkin method of weighted residuals to replace the current collocation method and conducting detailed tests on non-linear material profiles.

\section{References}

Balanis, C. A. (1989). Advanced Engineering Electromagnetics, John Wiley \& Sons, ISBN 0-47150316-9, New York

Becker, R.; Dobmann, G.; \& Rodner, C. (1988) Quantitative eddy current variants for micromagnetic microstructure multiparameter analysis 3MA. In: Review of Progress in Quantitative Non-Destructive Evaluation $7 B$, edited by Thompson, D.O. \& Chimenti, D. E. pp.1703 - 1707, Plenum Press, New York

Blitz, J. (1991). Electrical and Magnetic Methods of Non-destructive Testing, 1st edition, Adam Hilger, ISBN 0-7503-0148-1

Bowler, N. (2006). Frequency dependence of relative permeability in steel. In: Review of Progress in Quantitative Non-Destructive Evaluation, Vol. 25, edited by Thompson, D.O. \& Chimenti, D. E. pp.1269-1276, CP820, AIP, ISBN 0-7354-0312-0

Burden, R. L. Faires, J. D. (1989). Numerical Analysis, 4th Edition, Boston: PWS-KENT Publishing Company, ISBN: 0-534-98059-7

Cheng, C. C.; Dodd, C. V. \& Deeds, W. E. (1971). General Analysis of Probe Coils Near Stratified Conductors, International Journal of Non-destructive Testing, Vol. 3, pp. 109-130

Dodd, C. V. \& Deeds, W. E. (1968) 'Analytical Solutions to Eddy-Current Probe-Coil Problems' Journal of Applied Physics, Vol. 39, No. 6. pp 2829-2838

Glorieux C.; Moulder, J.; Basart, J. \& Thoen, J. (1999). The Determination of Electrical Conductivity Using Neural Network Inversion of Multi-frequency Eddy Current Probe Data, Journal of Physics D. Vol. 32, pp. 616-622

Harrison, D. J.; Jones, L. D. \& Burke, S. K. (1996). Benchmark problems for defect size and shape determination in eddy-current non-destructive evaluation, Journal of Non-Destructive Evaluation, Vol. 15, No. 1, pp. 21-34

Langhill, T. J. (1999). Painting Over Hot-Dip Galvanised Steel, Materials Performance, pp. 4449, December 1999

Moulder, J.; Uzal, E. \& Rose, J. H. (1992). Thickness and Conductivity of Metallic Layers from Eddy Current Measurements, Review of Scientific Instruments, Vol. 63, No. 6, pp 3455-3465.

Norton, S. J. \& Bowler, J. R. (1993). Theory of Eddy Current Inversion, Journal Of Applied Physics, Vol. 73, No. 2, pp. 501-512

Sadiku, M. N. O. (1992). Numerical Techniques in Electromagnetics, Florida: CRC Press, ISBN: 0-8493-4232-5

Stephenson, G. (1973). Mathematical Methods for Science Students' 2nd Edition, Published London Longman

Uzal, E.; Moulder, J. C.; Mitra, S. \& Rose, J. H. (1993). Impedance of Coils over Layered Metals with Continuously Variable Conductivity and Permeability: Theory and Experiment, Journal of Applied Physics, Vol. 74, No. 3, pp. 2076-2089 
Yildir, Y. B.; Klimpke, B. W. \& Zheng, D. (1992). A computer program for 2D/RS eddy current problem based on boundary element method, Available from:

http://www.integratedsoft.com/papers/techdocs/tech_2ox.pdf 


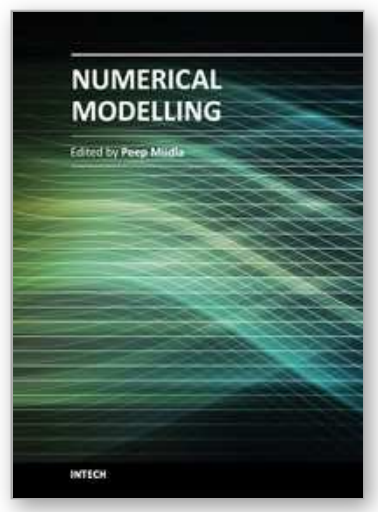

\author{
Numerical Modelling \\ Edited by Dr. Peep Miidla
}

ISBN 978-953-51-0219-9

Hard cover, 398 pages

Publisher InTech

Published online 23, March, 2012

Published in print edition March, 2012

This book demonstrates applications and case studies performed by experts for professionals and students in the field of technology, engineering, materials, decision making management and other industries in which mathematical modelling plays a role. Each chapter discusses an example and these are ranging from wellknown standards to novelty applications. Models are developed and analysed in details, authors carefully consider the procedure for constructing a mathematical replacement of phenomenon under consideration. For most of the cases this leads to the partial differential equations, for the solution of which numerical methods are necessary to use. The term Model is mainly understood as an ensemble of equations which describe the variables and interrelations of a physical system or process. Developments in computer technology and related software have provided numerous tools of increasing power for specialists in mathematical modelling. One finds a variety of these used to obtain the numerical results of the book.

\title{
How to reference
}

In order to correctly reference this scholarly work, feel free to copy and paste the following:

Philip May and Erping Zhou (2012). Numerical Modelling and Design of an Eddy Current Sensor, Numerical Modelling, Dr. Peep Miidla (Ed.), ISBN: 978-953-51-0219-9, InTech, Available from:

http://www.intechopen.com/books/numerical-modelling/numerical-modelling-and-design-of-an-eddy-currentsensor

\section{INTECH}

open science | open minds

\section{InTech Europe}

University Campus STeP Ri

Slavka Krautzeka 83/A

51000 Rijeka, Croatia

Phone: +385 (51) 770447

Fax: +385 (51) 686166

www.intechopen.com

\section{InTech China}

Unit 405, Office Block, Hotel Equatorial Shanghai

No.65, Yan An Road (West), Shanghai, 200040, China

中国上海市延安西路65号上海国际贵都大饭店办公楼 405 单元

Phone: +86-21-62489820

Fax: $+86-21-62489821$ 
(C) 2012 The Author(s). Licensee IntechOpen. This is an open access article distributed under the terms of the Creative Commons Attribution 3.0 License, which permits unrestricted use, distribution, and reproduction in any medium, provided the original work is properly cited. 